\title{
Influencia de la depresión, los estilos de comunicación y la adhesión al tratamiento sobre los niveles de glucosa en personas con diabetes
}

\author{
José Eduardo Rondón*, Irama Cardozo y Rosa Lacasella. \\ Instituto de Psicología, Universidad Central de Venezuela, Venezuela.
}

Recibido, agosto 17/2017

Concepto de evaluación, septiembre 21/2017

Aceptado, noviembre 7/2017

\begin{abstract}
Referencia: Rondón, J.E., Cardozo, I \& y Lacasella, R. (2018). Influencia de la depresión, los estilos de comunicación y la adhesión al tratamiento sobre los niveles de glucosa en personas con diabetes. Acta colombiana de Psicología, 21(2), 39-53. doi: http:// www.dx.doi.org/10.14718/ACP.2018.21.2.3
\end{abstract}

Resumen

\begin{abstract}
El objetivo de esta investigación fue analizar la influencia de la depresión, el estilo de comunicación (asertivo, pasivo o agresivo) y la adhesión al tratamiento sobre los niveles de glucosa en la sangre en sujetos con diabetes tipo 2. Por medio de un muestreo no probabilístico de tipo propositivo se seleccionó una muestra de 278 pacientes diagnosticados con diabetes tipo 2 (mínimo 1 año de diagnóstico), con edades entre 19 y 77 años, sin amputaciones ni comorbilidad con nefropatía o retinopatía diabética. Se utilizó un tipo de investigación no experimental, con un diseño transeccional-causal. Las relaciones fueron planteadas en un diseño de ruta basado en el análisis de regresión múltiple. Como instrumentos, se emplearon los Autoinformes de Actitudes y Valores en las Interacciones Sociales (ADCAS), el Cuestionario Tridimensional para la Depresión (CTD), y el Instrumento para Medir el Estilo de Vida en Diabéticos (IMEVID) -para evaluar la adhesión al tratamiento-, todos validados en población venezolana, además de la HbAlc - para medir el nivel de glucosa-. Los resultados muestran que las correlaciones entre las variables fueron bajas (con puntuaciones entre $r=-.12$ y $r=-.33 ; p<.05$ ), a excepción de dos, que pueden ser consideradas como moderadas: depresión y comunicación asertiva, con un $\mathrm{r}-.47, \mathrm{p}<.05$; y comunicación agresiva y comunicación asertiva, con un $\mathrm{r}=-.44, \mathrm{p}<.05$. Con el análisis posterior del modelo de regresión se observó que ninguna variable fue significativa para la predicción de los niveles de glucosa. En cuanto a la adhesión al tratamiento, la variable que más aporta a la predicción es la depresión. Y, con respecto al estilo de comunicación, se observó una correlación negativa, moderada y significativa entre depresión y asertividad. No se encontró relación entre la depresión y la comunicación agresiva ni pasiva. En conclusión, solo fueron corroboradas dos de las relaciones planteadas en el análisis de ruta. Se sugiere confirmar estos hallazgos en futuras investigaciones.
\end{abstract}

Palabras clave: depresión, estilos de comunicación, adhesión al tratamiento, diabetes mellitus tipo 2.

\section{The influence of depression, communication styles and treatment adherence on glucose levels in diabetics}

\begin{abstract}
The aim of the present research was to analyze the influence of depression, passive-assertive-aggressive communication styles and adherence to treatment over the glucose levels of subjects with Type 2 diabetes mellitus. Participants were 278 patients with a minimum of one year of having been diagnosed, selected through a purposive non-probability sampling, with ages ranging from 19 to 77 years old, with no amputations, nor comorbidity with nephropathy or diabetic retinopathy. A non-experimental investigation was developed through a cross-sectional design, establishing relationships between variables in a path analysis through multiple regression analyses. The instruments used were the Cuestionario Tridimensional para la Depresión (CTD, for its Spanish acronym) [Three-Dimensional Questionnaire for Depression]; the Autoinformes de Actitudes y Valores en las Interacciones Sociales (ADCAS, for its Spanish acronym) [Self Reports of Attitudes and Values in Social Interactions], and the Instrumento para Medir el Estilo de Vida en Diabéticos (IMEVID, for its Spanish acronym) [Instrument to Measure Diabetic Lifestyles] used to evaluate adherence to treatment. All of these instruments had been validated for the Venezuelan population. In addition, HbA1c was used to measure glucose level. Significant correlations between variables demonstrated
\end{abstract}

\footnotetext{
* Av. Neveri con calle Edison, Centro Comercial Los Chaguaramos, piso 2, ofic. 2-10, Caracas, Distrito Capital - Venezuela. +58 212 6052829, bernard85@gmail.com

Este estudio contó con el financiamiento y aval del Consejo de Desarrollo Científico y Humanístico de la Universidad Central de Venezuela.
} 
to be low (oscillating between $\mathrm{r}-0.12$ and $\mathrm{r}-0.33$ ), except for two of them considered to be moderate (depression-assertive communication $\mathrm{r}-0,47<\mathrm{p} 0,05$; and aggressive communication-assertive communication $\mathrm{r}-0,44<\mathrm{p} 0,05$ ). Likewise, from the path analysis, it could be seen that none of the variables were statistically significant for the prediction of glucose levels. In regards to treatment adherence, the variable that contributed the most to the prediction was depression. Concerning the communication styles, negative, moderate and significant associations were observed between depression and assertiveness. No relationship was found between depression and aggressiveness and passiveness. In conclusion, only two of the established relationships through the path analysis were corroborated. Confirmation of these findings is suggested for future investigations. Key words: Depression, communication styles, treatment continuance, diabetes mellitus type 2.

\title{
Influência da depressão, dos estilos de comunicação e da adesão ao tratamento sobre os níveis de glucose em personas com diabetes
}

\author{
Resumo
}

\begin{abstract}
O objetivo desta pesquisa foi analisar a influência da depressão, do estilo de comunicação (assertivo, passivo ou agressivo) e da adesão ao tratamento sobre os níveis de glucose no sangue em sujeitos com diabetes tipo 2. Por meio de uma amostragem não probabilística de tipo propositivo, foi selecionada uma amostra de 278 pacientes diagnosticados com diabetes tipo 2 (no mínimo um ano de diagnóstico), com idades entre 19 e 77 anos, sem amputações nem comorbidade com nefropatia ou retinopatia diabética. Utilizou-se um tipo de pesquisa não experimental com um desenho transversal-causal. As relações foram apresentadas em um desenho de rota baseado na análise de regressão múltipla. Como instrumentos, foram empregados os Autoinformes de Actitudes y Valores en las Interacciones Sociales (ADCAS), o Cuestionario Tridimensional para la Depresión (CTD) e o Instrumento para Medir el Estilo de Vida en Diabéticos (IMEVID) - para avaliar a adesão ao tratamento todos validados em população venezuelana, além da HbAlc — para medir o nível de glucose. Os resultados mostram que as correlações entre as variáveis foram baixas (com pontuações entre $r=-.12$ e $r=-.33 ; p<.05$ ), com exceção de duas, que podem ser consideradas como moderadas: depressão e comunicação assertiva, com um $\mathrm{r}-.47, \mathrm{p}<.05$; e comunicação agressiva e comunicação assertiva, com um $\mathrm{r}=-.44, \mathrm{p}<.05$. Com a análise posterior do modelo de regressão, observouse que nenhuma variável foi significativa para a previsão dos níveis de glucose. Quanto à adesão ao tratamento, a variável que mais contribui para a previsão é a depressão. E, com respeito ao estilo de comunicação, observou-se uma correlação negativa, moderada e significativa entre depressão e assertividade. Não foi encontrada nenhuma relação entre a depressão e a comunicação agressiva nem passiva. Em conclusão, apenas duas das relações apresentadas foram corroboradas na análise de rota. Sugere-se confirmar essas descobertas em pesquisas futuras.

Palavras-chave: adesão ao tratamento, depressão, diabetes mellitus tipo 2, estilos de comunicação.
\end{abstract}

\section{INTRODUCCIÓN}

La diabetes mellitus es una de las enfermedades crónicas no trasmisibles con mayor prevalencia en el mundo y constituye un factor de riesgo para el padecimiento de enfermedades cardiovasculares y complicaciones que incrementan el riesgo de mortalidad (Organización Mundial de la Salud, 2014). Específicamente, en Venezuela, según el Anuario de Mortalidad publicado por el Ministerio del Poder Popular para la Salud (2014), esta patología ocupaba el quinto lugar entre las 25 principales causas de muerte diagnosticadas en la población para el año 2012, además de que representaba la séptima causa de muerte para el grupo de sujetos fallecidos con edades entre los 25 y 44 años, la tercera causa en los fallecidos con edades entre los 45 y 74 años, y la cuarta causa de muerte de la población fallecida con más de 75 años.

El panorama es menos alentador si se revisan los informes de la Organización Mundial de la Salud (1999, en Pérez, Bautista \& Bazaldúa, 2008), según los cuales se espera que para el 2025 se duplicará el número de personas con diabetes en el mundo (de 120 a 240 millones de personas), lo que se estima ocurrirá en ciudades desarrolladas tanto por el incremento en la longevidad y el envejecimiento relativo de su población como por los estilos de vida basados en la alimentación inadecuada y el sedentarismo.

El diagnóstico de diabetes suele representar una fuente importante de estrés para el paciente, sobre todo por los cambios de hábitos que debe implementar para detener el avance de la enfermedad -además de su consecuente deterioro en la salud-, en donde el miedo y la incertidumbre son estados característicos. Esta modificación de la rutina se caracteriza por cambios en la alimentación, el chequeo constante de los niveles de glicemia y la realización de ejercicios físicos, entre otros comportamientos que pueden conllevar a la depresión o a alteraciones en las relaciones sociales, sobre todo por el hecho de tener que dejar de participar en actividades de índole social. Por tanto, las características de la enfermedad, vista como una fuente de estrés, hacen que el sujeto requiera de un proceso de adaptación a las actividades cotidianas, de la adquisición de información y nuevos conocimientos, y del 
desarrollo de habilidades con el fin de lograr una vivencia de la enfermedad sin dificultades (Oblitas, 2004; Ortiz \& Myers, 2014; Ortiz, Baeza-Rivera \& Myers, 2013; Ponce, Velázquez, Márquez, López \& Bellido, 2009).

Asimismo, las demandas relacionadas con el control de la diabetes, que involucran fuertes cambios en la dieta y en el ritmo de vida, además de la incorporación de nuevas rutinas, la reducción de ciertas actividades placenteras y las complicaciones la condición -como la retinopatía, la nefropatía, la cardiopatía, la neuropatía o las disfunciones sexuales, entre otras-, están relacionadas con una mayor tendencia a presentar depresión (Fabián, García \& Cobo, 2010). De igual forma, diversos estudios señalan que existe una frecuente comorbilidad entre la diabetes mellitus y la sintomatología depresiva (Constantino-Cerna, Bocanegra-Malca, León-Jiménez \& Díaz-Vélez, 2014; Doyle, Halaris \& Rao, 2014; Hermanns, Ehrmann \& Kulzer, 2015; Lyketsos, 2010; Molina, Acevedo, Yánez, Dávila \& González, 2013; Pineda et al., 2004).

A pesar de esto, la dirección de la relación entre diabetes y depresión aún no está clara, ya que se considera que la diabetes puede ser causa de la depresión por su impacto sobre la vida de la persona, pero, de igual manera, la presencia de depresión podría constituir un factor de riesgo para padecer diabetes (Colunga-Rodríguez, García, Salazar-Estrada \& Ángel-González, 2008; Dalzochio, Bonho, Feksa \& Berlese, 2014; Lyketsos, 2010).

Aunque los resultados no son concluyentes, distintos estudios (Campayo et al., 2010; Colunga-Rodríguez et al., 2008; Constantino-Cerna et al., 2014; De Jonge, et al., 2014; Young-Hyman, De Groot, Hill-Briggs, González, Hood \& Peyrot, 2016) dan cuenta de la comorbilidad de ambas condiciones, además de que indican que las personas con diabetes presentan un riesgo de padecer depresión dos o tres veces mayor que las personas sanas, donde dicho riesgo se asocia con factores como el tiempo de evolución de la enfermedad, el control sobre los comportamientos de autocuidado y las complicaciones de la diabetes. Sin embargo, Schmitt et al. (2015) señalan que altos niveles de depresión predicen un menor control de la glucosa y que esto podría relacionarse con la angustia asociada al hecho de padecer diabetes y no con la depresión per se, motivo por el cual resulta importante esclarecer esta relación.

Con respecto a la adhesión al tratamiento, Escandón, Azócar, Pérezy Matus (2015), así como Saur y Steffens (2010), señalan que la depresión tiene un impacto negativo sobre esta, sobre todo en pacientes con enfermedad crónica-como en el caso de la diabetes- $\mathrm{y}$ en conductas relacionas con la salud, como hacer ejercicio, dejar de fumar y controlar el peso; de manera que la comorbilidad de la diabetes con la depresión es percibida como una barrera para el desarrollo de conductas de autocuidado efectivas para el control de dicha enfermedad.
Asimismo, debido a que la depresión suele generar una alteración negativa en los hábitos de vida -alimentación, higiene y comportamientos sociales-, así como una expresión inadecuada de los sentimientos e ideas, la misma tiende a estar asociada a una menor adhesión al tratamiento y, por ende, a un menor control de la enfermedad (Jiménez \& Miguel-Tobal, 2003; Rodrigues, Egidio \& Cardoso, 2014; Young-Hyman et al., 2016). Y a esto se le suma la evidencia existente sobre la relación del déficit en habilidades sociales y un mayor riesgo o vulnerabilidad para padecer depresión (Santos, De Oliveira \& Sardinha, 2012).

Ahora bien, sabiendo que la persona con diabetes tiene que hacer frente a diversas presiones y "tentaciones" sociales, además de tener una comunicación constante con el personal de salud con la finalidad de cumplir el régimen de su tratamiento, es fundamental contar con un repertorio adecuado de destrezas sociales para afrontar dichas demandas positivamente. En este sentido, se ha planteado que los problemas de comunicación en la consulta médica, así como las dificultades en las relaciones con familiares y pares, representan un importante obstáculo para la adherencia al tratamiento y el control de la glucosa (Camacho, Lucero, Agazzi, Fernández \& Ferreira, 2013).

Al respecto, es necesario investigar el impacto de la participación de los pacientes en las decisiones sobre el manejo de su condición (Maidana et al., 2016), pero es más preciso estudiar en detalle el impacto del estilo de comunicación del paciente con sus pares y con el personal de salud sobre la adhesión al tratamiento con el fin de establecer pautas para la intervención adecuada.

En este sentido, Oliveira y Trujillo (2017) indican que es necesario educar no solo al paciente con diabetes, sino también a sus familiares y amigos en la realización de los comportamientos saludables requeridos para el control de la condición y la prevención de complicaciones. Y, teniendo en cuenta que en todas las interacciones sociales los estilos de comunicación que emplean los pacientes juegan un papel fundamental, su investigación se hace primordial tanto en la promoción de la salud como en la prevención de la enfermedad y su respectivo tratamiento.

$\mathrm{Al}$ respecto, se plantea que existen fundamentalmente tres estilos de comunicación: la comunicación asertiva, la pasiva y la agresiva. Una persona asertiva se comportaría de una manera intermedia entre los estilos pasivo y agresivo, al expresar de forma respetuosa, directa y honesta sus propias necesidades, deseos, opiniones, sentimientos y creencias; mientras que una persona agresiva lo haría de forma invasiva e irrespetuosa de los derechos del otro; y una pasiva, guardando opiniones para sí e irrespetando sus propios derechos (Ortega \& Calero, 2015; Stuart \& Laraia, 2006; Valls, 2009). 
Particularmente, una persona con un estilo de comunicación pasivo presentaría grandes dificultades para confrontar las presiones de los llamados a incumplir el tratamiento, pues carecería de las herramientas necesarias dirigidas a comunicar sus necesidades y limitaciones personales inherentes a la enfermedad. Mientras que una persona con un estilo de comunicación agresivo puede presentar un menor control de su diabetes, ya que se ha encontrado que tanto la preocupación como la expresión inadecuada de la ira pueden alterar los niveles de glucosa en sangre (Méndez \& Beléndez, 1994; Ramos \& Arnaud, 2011; Rodríguez \& García, 2013; Santos et al., 2012).

$\mathrm{Al}$ respecto, es posible asegurar que conocer las relaciones entre el estilo de comunicación, la adherencia al tratamiento y el control de la glucosa puede brindar valiosos aportes para mejorar el manejo y entender el impacto de las habilidades sociales del paciente con diabetes sobre el control de la enfermedad.

En relación con esto, en diversas investigaciones se ha demostrado que las personas que poseen mayor apoyo social tienden a presentar una mejor adhesión al tratamiento y un mejor control de la glucosa (Azzollini, Bail \& Vidal 2012; Gomes-Villas, Foss, Foss de Freitas \& Pace, 2012; Moral \& Cerda, 2015; Rondón, 2011); además de que las personas que emplean la búsqueda de apoyo como estrategia personal presentan una mayor adhesión al tratamiento (Garay, Santiago \& Malaraca, 2011), lo que está estrechamente relacionado con el empleo de estrategias de aproximación al problema que implican la búsqueda de apoyo social (Rondón \& Lugli, 2013).

Así, teniendo en cuenta lo expuesto anteriormente, podría esperarse que una persona que emplee un estilo de comunicación asertivo tendrá una mayor probabilidad de aproximarse a su problema de salud, ya que buscar y obtener un apoyo social favorecerá el establecimiento de redes sociales que coadyuven al manejo de su condición.

Adicionalmente, se ha encontrado que el cambio de estilo de vida para tener un buen control de la glucosa suele constituir una interferencia importante en el desarrollo de una adecuada adhesión a los tratamientos; al igual que padecer situaciones de estrés sostenido, trastornos psicológicos como la depresión, presentar sentimientos de inferioridad, vergüenza, desesperanza y tristeza, o, en general "sentirse mal" (Kaltman et al., 2016; Ramos \& Arnaud, 2011; Rodríguez \& García, 2013). Sin embargo, se han encontrado resultados contradictorios al respecto, ya que no hay acuerdo sobre si una baja adhesión al tratamiento farmacológico o no farmacológico podría traducirse en un mal control de la glucosa (Braz et al., 2012; Gomes-Villas et al., 2012; Rodríguez \& García, 2013); razón por la cual es necesario realizar estudios que permitan esclarecer la relación entre estas dos variables.
Ahora bien, considerando que la prevalencia de la depresión en pacientes con diabetes es tres veces mayor que en el resto de la población, es posible asegurar que este problema es de gran importancia y que debe ser considerado en el estudio de la adhesión al tratamiento de estos pacientes y su relación con el control adecuado de los niveles de glucosa. De igual manera, los estilos de comunicación pueden estar asociados con la depresión y con el manejo de los comportamientos implicados en la adhesión al tratamiento, por lo que el examen de dichas variables resulta de gran relevancia para esclarecer las relaciones entre los factores psicológicos y el padecimiento de la diabetes. Esto, además, servirá para generar programas de intervención que favorezcan el control de la condición y contribuyan con el incremento de la calidad de vida de los pacientes.

Con base en estos planteamientos, en la presente investigación se pretende analizar la influencia de la depresión, los estilos de comunicación (asertivo, pasivo y agresivo) y la adhesión al tratamiento sobre los niveles de glucosa en sangre ( $\mathrm{HbAlc}$ ) en sujetos con diabetes tipo 2. A partir de esto, en la Figura 1 se puede observar la hipótesis de la investigación, planteada en un modelo de ruta en el que aparecen las variables por estudiar y las posibles relaciones entre las mismas.

\section{MÉTODO}

\section{Diseño}

La presente investigación es de tipo no experimental, ya que no se posee control directo de las variables independientes (Kerlinger \& Lee, 2002); y el diseño empleado corresponde al transeccional-causal, puesto que se desea describir las relaciones causales entre variables en un momento determinado (Hernández, Fernández \& Baptista, 2006).

Específicamente, dado que se planteó un tipo de relación específico entre la depresión, los estilos de comunicación (pasivo, asertivo o agresivo), la adhesión al tratamiento y los niveles de glucosa, se empleó un "diseño de ruta", que puede ser visto como una extensión de la regresión múltiple, aunque se diferencia de esta en que trabaja con más de una variable dependiente y en que plantea relaciones del tipo "X" causa "Y" y "Y" causa "Z" (Angelucci, 2007).

\section{Participantes}

Por medio de un muestreo no probabilístico de tipo propositivo (Kerlinger \& Lee, 2002), se seleccionaron 278 pacientes con diagnóstico de diabetes tipo 2 -con mínimo un año con dicho diagnóstico-, entre hombres y mujeres -con edades entre los 19 y 77 años- que no presentaban comorbilidad con nefropatía diabética (en diálisis) o retinopatía 


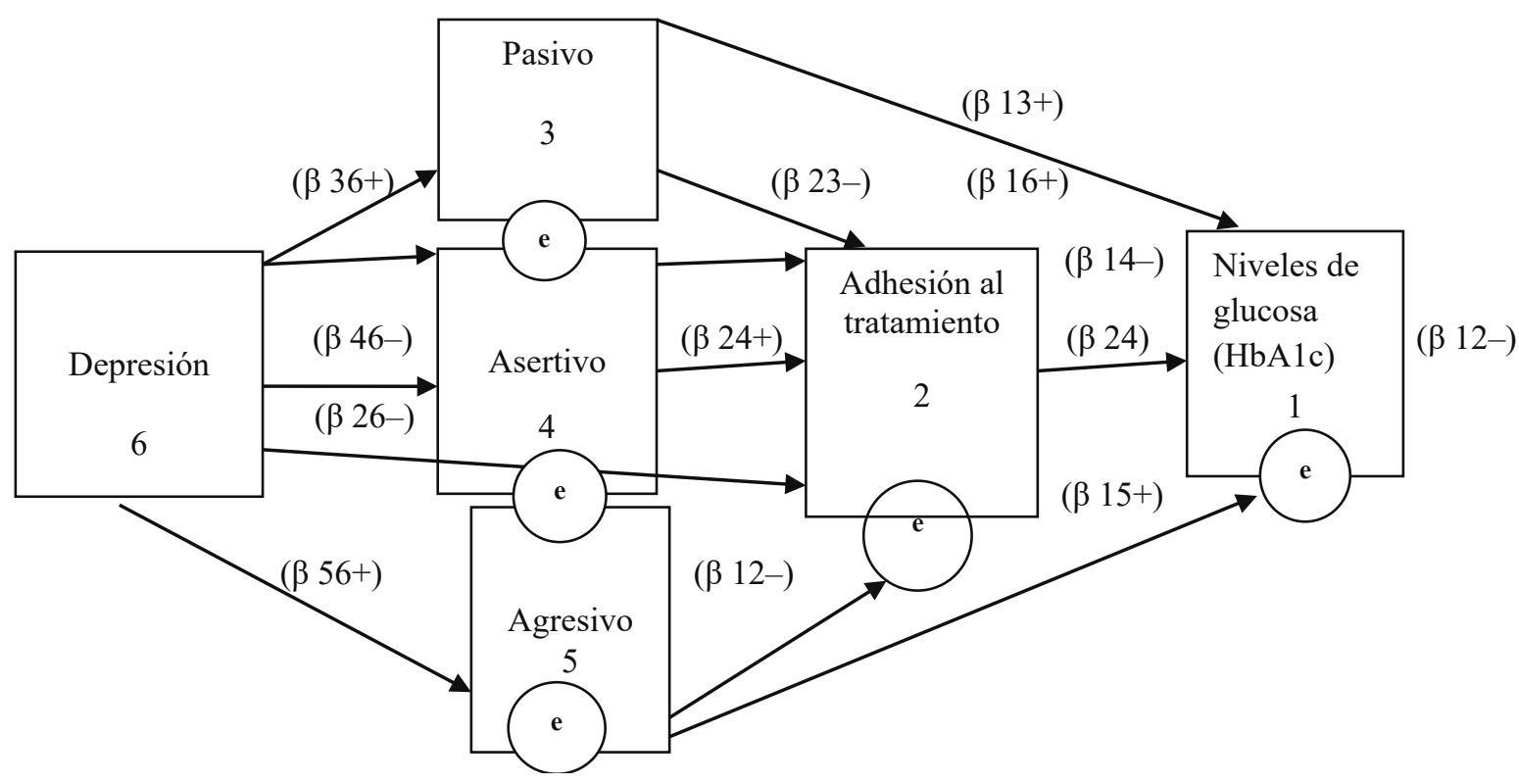

Figura 1. Diagrama de ruta propuesto. Cada flecha representa la relación asimétrica propuesta entre las dos variables. Los coeficientes beta indican la magnitud y dirección del efecto (positiva o negativa) y los números indican las variables implicadas. "e" representa el término de "error".

diabética (ceguera), ni amputaciones de miembro alguno. A continuación (véase Tabla 1), se detallan algunos datos sociodemográficos de la muestra estudiada.

Tabla 1

Datos sociodemográficos de los participantes

\begin{tabular}{lc}
\hline & Participantes \\
\hline Mujeres & 159 \\
Hombres & 119 \\
Edad media & 59 años \\
Nivel medio de instrucción & Bachillerato completo \\
Tiempo medio con la condición & 10 años \\
\hline
\end{tabular}

\section{Instrumentos}

Cuestionario Tridimensional para la Depresión (CTD).

Este instrumento fue desarrollado por Jiménez y MiguelTobal (2003) para evaluar la depresión desde el modelo teórico de los tres sistemas de respuesta (cognitivo, fisiológico y motor). La versión original cuenta con cuatro factores que explican el $61.41 \%$ de la varianza total, tiene una confiabilidad test retest de .56 y un alfa de Cronbach para la población general de .92 .

Específicamente, se empleó la adaptación venezolana en pacientes con enfermedades crónicas realizada por Cardozo, Guarino y Rondón (2016), que cuenta con 32 ítems divididos en tres dimensiones: 11 en la escala motriz, 12 en la cognitiva y 9 en la fisiológica. Con respecto a la escala de respuesta, las opciones para cada ítem van del
0 al 4, donde 0 significa "casi nunca" y 4 , "casi siempre". Para la calificación total, las puntuaciones van de 0 (bajo nivel de depresión) a 128 (alto nivel de depresión).

Con respecto a los índices de confiabilidad, todas las escalas fueron superiores a .75 , el índice de consistencia interna de la prueba total es de .85 , las correlaciones entre escalas oscilan entre .37 y .49 , y las correlaciones entre las tres escalas y la prueba total se encuentran entre .70 y .81 . Con respecto a la validez de la prueba, se exploró la validez de criterio a partir del cálculo del índice de correlación con el Inventario de Ansiedad Rasgo (IDARE), dada la relación entre depresión y ansiedad ampliamente reportada en la literatura (Agudelo, Buela-Casal \& Spolberger, 2007; Agudelo, Casadiegos \& Sánchez, 2009; Moscoso, 2014; Páez, Jofré, Azpiroz \& De Bortoli, 2009), donde se encontró un índice de correlación .70. Finalmente, con respecto a la validez estructural, los análisis demuestran que el cuestionario presenta una estructura factorial que explica el $31.94 \%$ de la varianza total.

Autoinformes de Actitudes y Valores en las Interacciones Sociales (ADCAS). Este cuestionario fue creado en 1992 por E. Manuel García Pérez y Ángela Magaz Lago con la finalidad de identificar las actitudes y el sistema de valores del sujeto con respecto a las relaciones sociales, además de establecer el perfil de sus actitudes y valores en las interacciones sociales: pasivos, agresivos, asertivos y 
pasivo-agresivos (García \& Magaz, 2000). Específicamente, evalúa las dimensiones de autoasertividad, que es el grado o nivel de respeto y consideración hacia los sentimientos, ideas y comportamientos propios; y de heteroasertividad, que se refiere al grado o nivel de respeto y consideración hacia los sentimientos, ideas y comportamientos ajenos.

Según los autores, la dimensión de autoasertividad posee un alfa de Cronbach de .90, mientras que la heteroasertividad, un alfa de .85. Asimismo, la prueba posee una validez discriminante mayor a $75 \%$ al comparar los resultados del test con el criterio de psicólogos especializados en el área. Diferentes combinaciones de ambas dimensiones generan los cuatro estilos de comunicación (García \& Magaz, 2000).

Específicamente, se empleó la adaptación en población venezolana con enfermedades crónicas realizada por Rondón, Cardozo y Lacasella (2014), en donde, al igual que en la versión original, aparecen cuatro opciones de respuesta: "nunca o casi nunca", "a veces", "a menudo" y "siempre o casi siempre"; y se reporta para la prueba en su totalidad un índice de consistencia interna (alfa de Cronbach) de .92, así como para la dimensión de heteroasertividad, de .89 (14 ítems), y para la dimensión de autoasertividad, de 85 (19 ítems). Los dos factores explican $35.20 \%$ de la varianza.

Instrumento para Medir el Estilo de Vida en Diabéticos (IMEVID), adherencia al tratamiento. El IMEVID es un instrumento de autoadministración elaborado por LópezCarmona, Arisa-Andraca, Rodríguez-Moctezuma y MunguiaMiranda (2003) para medir adhesión al tratamiento como estilo de vida en pacientes con diabetes mellitus tipo 2 . Según los autores, el alfa de Cronbach para la calificación total del instrumento es de .81, y el coeficiente de correlación test-retest es de .84, el cual explica con ocho factores el $60.4 \%$ de la varianza total.

Para el presente estudio se empleó la adaptación en población venezolana con enfermedades crónicas realizada por Rondón et al. (2014), en donde, al igual que en la versión original, se responde asignando calificaciones de 0,2 y 4 a las tres opciones de respuesta, donde 4 corresponde a la conducta deseable. En la presente adaptación se establece una nueva estructura factorial con ocho factores que explican $59.56 \%$ de la varianza. Además, la prueba en su totalidad posee un índice de consistencia interna (alfa de Cronbach) de .60 , y, específicamente, de .72 en el factor de adhesión (3 ítems), .92 en el de consumo de alcohol (2 ítems), .97 en el de consumo de tabaco ( 2 ítems), .48 en el de tipo de nutrición (4 ítems), .34 en el de emoción (3 ítems), 22 en el de ejercicio (4 ítems), .29 en el de información ( 2 ítems) y .29 en el de cantidad de comida (3 ítems). A mayor puntuación, mayor adhesión al tratamiento.
Niveles de glucosa. Se evaluaron los niveles de glucosa a partir del parámetro hemoglobina glicosilada $(\mathrm{HbAlc})$, que consiste en un análisis de la sangre para dar cuenta de los niveles medios de glucosa en los tres meses previos a la medición en los pacientes diabéticos-se obtuvo el reporte escrito del resultado de laboratorio del último mes de cada paciente-. Esta prueba mide la cantidad de glucosa que se ha adherido a los glóbulos rojos y otras células, puesto que existe una relación directa entre dichos valores y la aparición de complicaciones microvasculares en estos pacientes (Ricard, 2008). Según la Asociación Latinoamericana de Diabetes (2013), la HbA1c es el indicador más idóneo para conocer el control de glucosa, donde un parámetro de $\mathrm{HbA} 1 \mathrm{c}$ menor a siete es considerado como adecuado en una persona con diabetes.

\section{Procedimiento}

Se llevaron a cabo entrevistas a 500 personas, entre hombres y mujeres, diagnosticadas con diabetes mellitus tipo 2 que asistían a distintos centros de salud de la ciudad de Caracas. Del total de entrevistados, se seleccionaron 278 sujetos que cumplían con los criterios señalados anteriormente. A continuación, se les indicó el propósito de la investigación y se les solicitó, además de su marcador de hemoglobina glicosilada con una antigüedad no mayor a un mes, su consentimiento de participación por escrito, con el fin de cumplir con los parámetros éticos de la investigación.

La aplicación de los cuestionarios fue en forma de entrevista tanto de manera grupal como individual y fue llevada a cabo por estudiantes del último semestre de Psicología (previamente entrenados). La recolección de los datos duró aproximadamente nueve meses, de enero a septiembre de 2015. Completada la recolección de datos, se continuó con su codificación y vaciado en el paquete estadístico SPSS 19, con la finalidad de realizar los análisis estadísticos pertinentes y así dar respuesta al objetivo principal de esta investigación.

Se debe mencionar que para la realización de este estudio se contó con el financiamiento y aval del Consejo de Desarrollo Científico y Humanístico de la Universidad Central de Venezuela, y que no existe conflicto de intereses entre los autores.

\section{Análisis de datos}

Con el propósito de describir el comportamiento de las variables en estudio y de cumplir los supuestos requeridos para obtener las regresiones múltiples -y de esta forma ejecutar el análisis de ruta (path analysis)-, se calcularon los estadísticos descriptivos tanto de tendencia central, dispersión y demás características de la distribución de las variables. Posteriormente, 
mediante el coeficiente $r$ de Pearson, se hallaron las correlaciones entre las variables propuestas; $y$, por último, se llevó a cabo el análisis de ruta, calculando las regresiones múltiples con el método "paso a paso" para cada variable endógena del modelo planteado (estilo pasivo, estilo asertivo, estilo agresivo, adhesión al tratamiento y niveles de glucosa).

Finalmente, se obtuvieron los coeficientes de correlación múltiple, el coeficiente de determinación y los coeficientes b y $ß$ para cada una de las variables predictoras involucradas en el modelo de regresión, y se contrastó el modelo propuesto con el modelo resultante.

\section{Consideraciones éticas}

Durante todo el proceso del estudio se cumplió con el principio de confidencialidad por medio del anonimato de los datos, y al final del estudio se realizó una devolución de información general a los directivos de las instituciones de salud donde se recopilaron los datos.

\section{RESULTADOS}

Acontinuación, se presentan los estadísticos descriptivos de las variables en estudio, su respectivo análisis correlaciones y, por último, el análisis de ruta para responder al objetivo de investigación.

\section{Descripción de las variables}

Con la finalidad de describir las variables depresión, adhesión al tratamiento y niveles de glucosa en la sangre (HbAlc) en los participantes de la investigación, además de garantizar el supuesto de distribución normal de las variables, a continuación se presentan los análisis de los estadísticos descriptivos de tendencia central (media, mediana y moda), dispersión (desviación típica, mínimo y máximo) y las características de la distribución (asimetría y curtosis). Cabe destacar que los estilos de comunicación pasivo, asertivo y agresivo, por ser variables que se desplazan en un continuo dicotómico, no se incluyeron en este análisis (véase Tabla 2).

Los estadísticos presentados anteriormente muestran que las variables estudiadas tienden a distribuirse de forma normal, y que presentan leves distorsiones significativas según el estadístico de K-Smirnov-asociado a una significación de .05-. Sin embargo, se debe destacar que esta prueba es muy sensible a las asimetrías, por lo cual se prosigue con el análisis.

\section{Asociación de las variables}

Con el propósito de garantizar el supuesto de baja multicolinealidad entre las variables y realizar el análisis de ruta, se calcularon las correlaciones bivariadas, que debían ser de valores bajos. En la Tabla 3 se exponen las correlaciones entre las variables en estudio, donde se observa que todas las correlaciones son bajas, a excepción de dos, que son moderadas: depresión-comunicación asertiva y comunicación agresiva-comunicación asertiva.

Tabla 2

Estadísticos descriptivos de las variables en estudio

\begin{tabular}{ccccccccc}
\hline Variables & Media & \multirow{2}{*}{ Mediana } & Moda & $\begin{array}{c}\text { Desv. } \\
\text { Típica }\end{array}$ & Asimetría & Curtosis & Mínimo & Máximo \\
\hline HbA1c & 7.74 & 7.5 & 6 & 2.05 & .82 & .75 & 4.07 & 15.4 \\
Adhesión al tratamiento & 56 & 56 & 55 & 5.34 & -.51 & .03 & 39 & 67 \\
Depresión & 29 & 25 & 9 & 19.28 & 1.08 & 1.21 & 0 & 98 \\
\hline
\end{tabular}

Tabla 3

Correlaciones entre las variables de estudio

\begin{tabular}{cccccc}
\hline Variables & \multirow{2}{*}{ Depresión } & $\begin{array}{c}\text { Adhesión al } \\
\text { Tratamiento }\end{array}$ & $\begin{array}{c}\text { Com. } \\
\text { Agresiva }\end{array}$ & $\begin{array}{c}\text { Com. } \\
\text { Asertiva }\end{array}$ & Com. Pasiva \\
\hline HbA1c & $\mathbf{. 1 4}$ &.$- \mathbf{1 4}$ & .01 & $\mathbf{- . 1 2}$ & -.5 \\
Depresión & & $\mathbf{- . 3 3}$ & 0 & $\mathbf{- . 4 7}$ & .04 \\
Adhesión al tratamiento & & & 0 & $\mathbf{. 2 2}$ & .04 \\
Com. Agresiva & & & & -.44 & -.1 \\
Com. Asertiva & & & & & -.2 \\
\hline
\end{tabular}

Nota. En negrilla p <.05; Comunicación Agresiva: No (0) Sí (1); Comunicación Asertiva: No (0) Sí (1); Comunicación Pasiva: No (0) Sí (1). 
Análisis de ruta (path analysis)

Con el objetivo de verificar las relaciones entre las variables en estudio, se procedió a realizar el análisis de ruta (path analysis), tomando como nivel de significación un alfa de.05. Así, se obtuvieron los coeficientes de correlación múltiple, el coeficiente de determinación y los coeficientes $b$ y $\beta$ para cada una de las variables predictoras involucradas en el modelo de regresión.

Niveles de glucosa (hemoglobina glicosilada- HbAlc)

En la Tabla 4 se aprecia una correlación múltiple baja y positiva (.20) con la mejor combinación lineal entre las variables adhesión al tratamiento, asertividad, agresividad, pasividad y depresión. El coeficiente de determinación ajustado fue de .02, es decir, que el modelo explica el $2 \%$ de la varianza total de manera no significativa $(F=2.22$; $g l=5 ; p>.05)$. Además, al evaluar - por medio del coeficiente $\beta$ - las magnitudes del efecto directo de cada una de las variables (por separado) sobre los niveles de glucosa (HbAlc), se encontró que ninguna de las variables fue significativa en su predicción.

\section{Adhesión al tratamiento}

La Tabla 5 muestra una correlación múltiple moderada y positiva (.35) con la mejor combinación lineal de las variables asertividad, agresividad, pasividad y depresión. El coeficiente de determinación ajustado fue de .11, es decir, que el modelo explica el $11 \%$ de la varianza total de manera significativa $(F=9.34 ; g l=4 ; p=.000)$. Al evaluar -por medio del coeficiente $\beta$ - las magnitudes del efecto directo de cada una de las variables por separado sobre la adhesión al tratamiento, se encontró que la variable que más aporta a la predicción es la depresión, con una correlación negativa, baja y significativa $(\beta=-.29 p<.05)$. Las demás variables incluidas en el modelo no tuvieron por sí solas un efecto significativo en la predicción de la adhesión al tratamiento.

\section{Asertividad, agresividad y pasividad}

En la Tabla 6 se observa una correlación múltiple moderada y positiva (.48) entre la asertividad y la depresión. El coeficiente de determinación ajustado fue de .22, es decir, que el modelo explica el $22 \%$ de la varianza total de manera significativa $(F=81.1 ; g l=1 ; p=.000)$. Al evaluar - por medio del coeficiente $\beta$ - las magnitudes del efecto directo, se encontró que la depresión presenta una correlación negativa, moderada y significativa $(\beta=-.48$ $p<.05)$ con la asertividad.

Además, en la predicción de la variable agresividad no se observa correlación entre esta ( 0 ) y la depresión, donde el coeficiente de determinación ajustado fue de 0 , lo que quiere

Tabla 4

Resumen del modelo y coeficientes B y Beta para la variable niveles de glucosa (HbAlc)

\begin{tabular}{|c|c|c|c|c|c|}
\hline $\mathrm{R}$ & R 2 & R 2 Corregida & ET & Durwin Watson & \\
\hline \multirow[t]{3}{*}{.2} & .04 & .02 & 2.03 & 1.8 & \\
\hline & Coeficie & Estandarizados & $\begin{array}{c}\text { Coeficiente } \\
\text { Estandarizado }\end{array}$ & & \\
\hline & B & E. T & Beta & $\mathrm{t}$ & $\mathrm{p}$ \\
\hline Adhesión & -.04 & .02 & -.10 & -1.50 & .13 \\
\hline Agresividad & -.24 & .37 & -.05 & -.65 & .51 \\
\hline Asertividad & -.45 & .34 & -.11 & -1.32 & .19 \\
\hline Depresión & .01 & .01 & .06 & .88 & .38 \\
\hline Pasividad & -.74 & .61 & -.08 & -1.21 & .23 \\
\hline
\end{tabular}

Tabla 5

Resumen del modelo y coeficientes B y Beta para la variable adhesión al tratamiento

\begin{tabular}{|c|c|c|c|c|c|}
\hline $\mathrm{R}$ & R 2 & R 2 Corregida & ET & Durwin Watson & \\
\hline \multirow[t]{4}{*}{.35} & .12 & .11 & 5.05 & 1.8 & \\
\hline & & & Coeficiente & & \\
\hline & Coeficie & Estandarizados & Estandarizado & & \\
\hline & B & E. $\mathrm{T}$ & Beta & $\mathrm{t}$ & $\mathrm{p}$ \\
\hline Agresividad & -.31 & .92 & -.02 & -.34 & .74 \\
\hline Asertividad & .96 & .84 & .09 & 1.14 & .25 \\
\hline Depresión & -.08 & .02 & -.29 & -4.31 & .00 \\
\hline Pasividad & 1.72 & 1.51 & .07 & 1.14 & .26 \\
\hline
\end{tabular}


Tabla 6

Resumen del modelo y coeficientes B y Beta para las variables asertividad, agresividad, pasividad

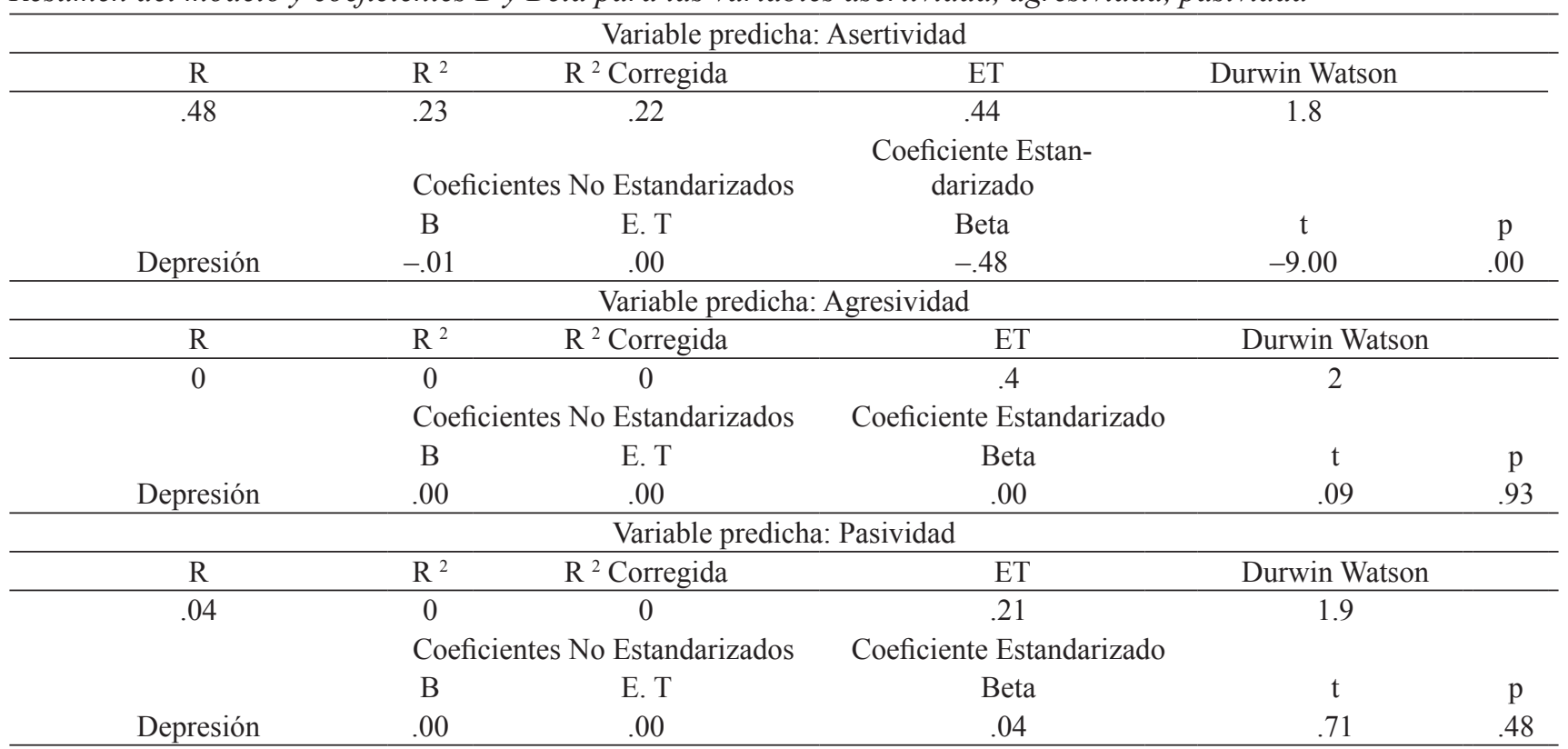

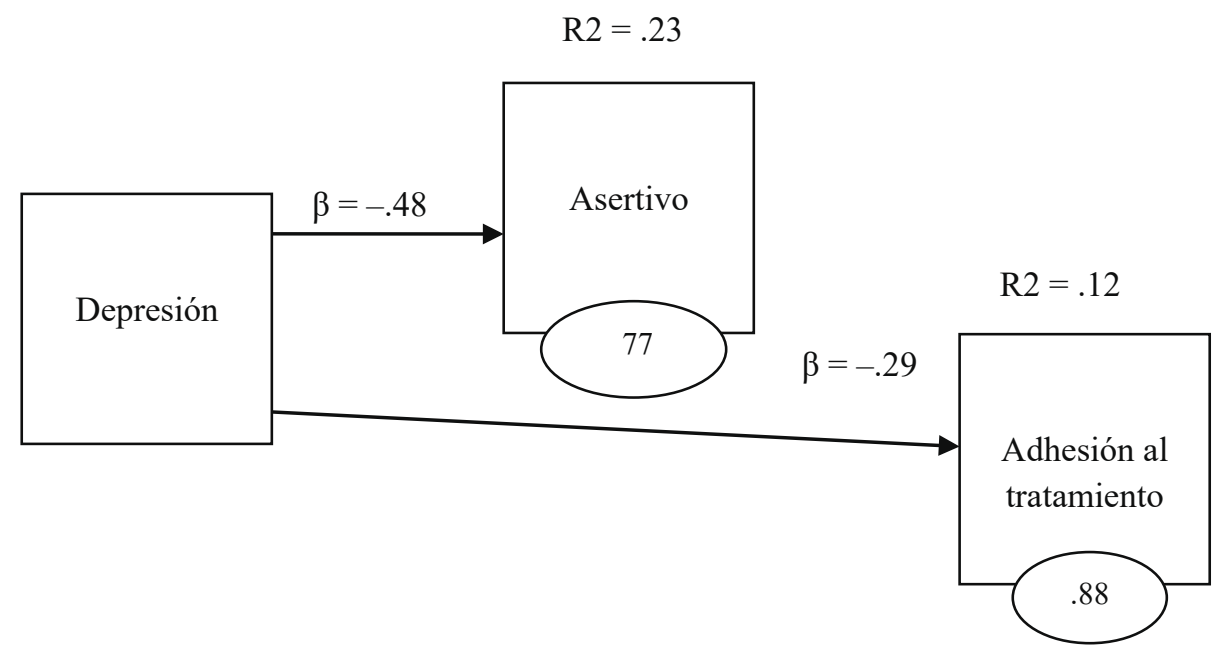

Figura 2. Diagrama de ruta resultante.

decir que el modelo no explica la varianza. Lo mismo que en la predicción de la variable pasividad, pues tampoco se observa correlación entre esta (.04) y la depresión, donde el coeficiente de determinación ajustado fue de 0 , lo que quiere decir que el modelo tampoco explica la varianza.

En conclusión, solamente dos de las relaciones planteadas fueron corroboradas, lo que dio origen al siguiente modelo (véase Figura 2).

\section{DISCUSIÓN}

Sobre la base de los resultados obtenidos, y para dar cuenta de la investigación realizada, sería conveniente recordar el objetivo principal de esta indagación, que fue "analizar la influencia de la depresión, el estilo de comunicación (asertivo pasivo o agresivo) y la adhesión al tratamiento sobre los niveles de glucosa en sangre 
(hemoglobina glicosilada-HbA1c-) en sujetos con diabetes tipo 2, proponiendo para este análisis, un modelo de ruta".

\section{Comportamiento de indicadores descriptivos de la muestra bajo estudio}

Sexo.

La mayoría de los participantes de la investigación fue del sexo femenino. Esto concuerda con autores tales como Calderón, Vargas y Lozano (2015), y Rondón y Lugli (2013), quienes revelan una mayor prevalencia de diabetes mellitus tipo 2 en personas del sexo femenino; tal prevalencia es avalada por lo reportado en el Anuario de Mortalidad 2012 en Venezuela.

\section{Edad.}

El promedio de edad de esta muestra se encuentra alrededor de los 59 años, lo que coincide con otras investigaciones (Camejo, García, Rodríguez, Carrizales \& Chique, 2012; Organización Panamericana de la Salud, 2016; Patilla, 2011; Rondón, 2015; Rondón \& Lugli, 2013), en las cuales se reporta que el diagnóstico de la diabetes mellitus tipo 2 se da con frecuencia en la edad madura (posterior a los 45 años). Cabe destacar que estos resultados están empezando a cambiar en el ámbito latinoamericano, ya que, según el Informe Nacional de Estadísticas de la Diabetes (Centros para el Control y la Prevención de Enfermedades, 2014) y el estudio de Aguilar-Salinas, Gómez y Gómez (2011), en México, por ejemplo, la edad promedio de diagnóstico de la enfermedad está disminuyendo y se encuentran con cierta frecuencia en menores de 40 años. Por tanto, será conveniente en estudios ulteriores considerar esta variable.

\section{Nivel de instrucción.}

La mayor parte de los participantes de la presente investigación revela un nivel de instrucción de bachillerato completo. Según las investigaciones de Rondón y Lugli (2013) y Rondón (2015), realizadas en Venezuela, el nivel instruccional de las muestras empleadas se sitúa principalmente en este rango. Este factor es importante porque explicaría, en parte, que personas con menor nivel instruccional tienden a reportar más enfermedades, dado que no pueden ponderar a cabalidad la vulnerabilidad biológica 0 social a la que están expuestos (De la Cruz-Sánchez, Feu \& Vizuete-Carrizona, 2012). Cabe destacar que, en Venezuela, según reporte del Instituto Nacional de Estadísticas (2014), la cantidad de personas alfabetizadas es cada vez mayor, lo cual podría indicar, aunque no garantizar, un nivel de instrucción más elevado que, a su vez, tenga una mejor ponderación de los riesgos que significan desestimar el cuidado que se debe tener con respecto a la diabetes.

\section{Análisis de las variables de estudio}

Niveles de glucosa (Hemoglobina glicosilada- HbAlc). El promedio de hemoglobina glicosilada fue de 7.74. Este indicador, aun estando un poco por encima de los parámetros estimados como normales $(7 \%$, de acuerdo con la Asociación Latinoamericana de Diabetes, 2013), muestra un buen ajuste de esta condición orgánica.

\section{Adhesión al tratamiento.}

Según la media de calificaciones obtenidas para la medición de la adhesión al tratamiento, que se situó en 55, se puede afirmar que en esta muestra en particular se aprecia un nivel bastante aceptable de adhesión al tratamiento. Este resultado avala en forma indirecta los resultados obtenidos por Patilla (2011) y los de Marín y Mubayad (2012), en los que se resalta la importancia de la adhesión al tratamiento no solo en cuanto al seguimiento de las prescripciones médicas -que consecuentemente tendrán incidencia en el control de la diabetes como tal-, sino a la posibilidad de disminuir los niveles de ansiedad y depresión asociados comúnmente a esta condición.

\section{Depresión.}

En esta muestra, se observan niveles depresivos bajos, así como una diferencia muy marcada entre las medidas de tendencia central; lo cual indica una distribución con marcada asimetría. Resultados similares se encontraron en las investigaciones de Patilla (2011), Marín y Mubayad (2012), Benítez et al. (2011) con respecto a los niveles de depresión observados en sus muestras respectivas.

\section{Análisis de las asociaciones entre las variables}

Un análisis de todas las posibles asociaciones que podían establecerse entre las variables consideradas arrojó dos resultados significativos: la relación entre la depresión y la comunicación asertiva, y la relación entre comunicación asertiva y la comunicación agresiva.

Con respecto a la asociación entre la comunicación asertiva y la comunicación agresiva, los hallazgos demuestran que mayor asertividad se relaciona con menor agresividad, y esto tiene sentido en tanto la conducta asertiva es una habilidad que se caracteriza por la expresión adecuada y sincera de los sentimientos, ideas, opiniones y deseos propios, así como por el respeto propio y hacia los otros, lo cual es incompatible con el comportamiento agresivo (Castaños, Reyes, Rivera \& Díaz, 2011; García \& Magaz, 
2000; Martínez, Justicia \& Fernández, 2016; Valls, 2009). Al respecto, la asertividad es el punto medio en el espectro continuo entre la conducta pasiva y la agresiva, por lo que la conducta asertiva transmite sensación de autoconfianza y de respeto por la otra persona, así como el hecho de hablar con claridad y tener la libertad de rechazar una proposición no razonable, además de compartir su criterio con el otro (Ortega \& Calero, 2015; Stuart \& Laraia, 2006).

Y en cuanto a la asociación entre depresión y comunicación asertiva, se ha encontrado evidencia al respecto en otros estudios (Arancibia, Behar, Marín, Inzunza \& Madrid, 2016; Patilla, 2011), donde es común encontrar que la depresión genera una alteración en los hábitos de vida, alimentación e higiene, así como un decremento de comportamientos sociales; lo que puede favorecer la expresión inadecuada de los sentimientos e ideas $y$, consecuentemente, provocar una menor adhesión al tratamiento y un menor control de la condición (Jiménez \& Miguel-Tobal, 2003; Rodrígues et al., 2014). A esto se le suma que existe evidencia sobre la presencia de déficit en habilidades sociales, el cual implica un mayor riesgo o vulnerabilidad para padecer depresión (Santos et al., 2012).

Además, una persona de conductas pasivas frente a la condición de la diabetes podría manifestar dificultades para desafiar la presión social, dado que carece de ciertas destrezas que le permitan abordar sus necesidades comunicacionales en forma apropiada $y$, por tanto, tratar adecuadamente su condición. Igualmente, la expresión inadecuada de la ira por parte de estos pacientes podría alterar los niveles de glucosa en sangre, por lo que un estilo de comunicación asertivo favorecería la disminución o, por lo menos, el mantenimiento al mínimo de estos niveles. El estudio y examen de estas relaciones puede ser un aliado poderoso para el manejo de las habilidades sociales del paciente con diabetes (Méndez \& Beléndez, 1994; Ramos \& Arnaud, 2011; Rodríguez \& García, 2013; Santos et al., 2012) y, por ende, del mejoramiento de su calidad de vida.

\section{Análisis de ruta (path analysis)}

Al comprobar las relaciones que se establecen entre las variables en estudio - por medio de un modelo de análisis de ruta (path analysis) y tomando como nivel de significación un alfa de .05-, se encontró que las variables con mayor peso relacional, asociativo y predictor fueron la depresión, la adhesión al tratamiento y el estilo comunicacional asertivo, de la forma en que se expone a continuación.

La depresión afecta de manera moderada, negativa y significativa al estilo comunicacional asertivo.

Esto quiere decir que a medida que las personas presentan menores niveles de depresión, tendrán un repertorio social comunicacional más adecuado, con el cual harán énfasis en la defensa de sus derechos a tener y conservar una mejor calidad de vida, así como mayor disciplina con los hábitos alimentarios, los ejercicios, y que la comunidad respete sus decisiones (Ledón, 2014; Patilla, 2011). A lo largo de su experiencia con la condición, las personas van desarrollando diferentes sintomatologías, especialmente, ansiedad y depresión (Ledón, 2012; Nagel, Azócar, Pérez \& Matus 2015), y es precisamente mediante el entrenamiento de las destrezas sociales que se han encontrado mejoras en su calidad de vida.

Al respecto, el reto que deben afrontar estas personas es la forma adecuada para comunicar a sus seres queridos sus necesidades, así como sus temores en cuanto al cambio biológico que se está dando, además de la manera en que van a enfrentar esos cambios (cardiovasculares, gastrointestinales, dermatológicos), las dudas, las dificultades de la reconstrucción de su imagen corporal, la autoestima y el sentido general de la vida (Ledón, 2012).

Incluso, la relación con familiares y amigos (cuidadores primarios) se puede ver afectada por las posibles complicaciones y los cambios necesarios en el estilo de vida, puesto que aparecen desacuerdos o discusiones que conllevan al desgaste físico, económico y emocional. Por ejemplo, los cambios en la alimentación y otras modificaciones en el estilo de vida del paciente pueden generar una resistencia al cambio en el resto de los integrantes de la familia y afectar su adhesión al tratamiento. Esto se atribuye a una falta de empatía y a la poca conciencia sobre la condición que tiene tanto el paciente como su familia. Por ello, se enfatiza en la importancia del abordaje de los repertorios sociales con la finalidad de que las personas que posean esta condición se hagan responsables de sus propios procesos de salud, y, en consecuencia, puedan dirimir de manera adecuada las exigencias de la misma.

La depresión afecta de manera baja, negativa y significativa a la adhesión al tratamiento.

El modelo de indefensión propuesto por Seligman (1975) establece que una persona que se encuentra en este estado depresivo se caracteriza por la disminución de su tasa de respuesta; en este sentido, la adhesión al tratamiento implica un conjunto de conductas que se ven disminuidas en una persona con dicho trastorno. La relación encontrada se corrobora con la mayoría de las investigaciones consultadas en el área (González et al., 2015; Kaltman et al., 2016; Marín \& Mubayed, 2012; Moral \& Cerda, 2015; Patilla, 2011; Sánchez-Cruz, Hipólito-Lóenzo, Mugartegui-Sánchez \& Yánez-González, 2016), en las cuales se destaca que las personas con depresión tienen un menor seguimiento de las 
prescripciones médicas, además de no adhesión al tratamiento indicado, pobre control de la glucosa, $\mathrm{y}$, consecuentemente, a un deterioro progresivo no solo de la salud física como tal, sino de su salud psicológica y su calidad de vida. Cabe destacar que existe una diferencia entre el seguimiento de prescripciones médicas y la adhesión al tratamiento, en el sentido de que este último es un conjunto mayor que integra otros aspectos relevantes, como la alimentación y el ejercicio físico.

El triángulo conformado por la medicación, la nutrición y la ejercitación (Marco, 2014) es el pilar fundamental para que la persona con una condición diabética pueda desplegar un nivel adecuado de bienestar que le permita tener relaciones sociales, familiares, laborales, personales óptimas, además de con un control apropiado de la glucosa y la prevención de complicaciones, que, eventualmente, se reflejarán en otros indicadores de salud biológica y en una calidad de vida acorde con los estándares sociales (González et al., 2015).

En el caso de los pacientes diabéticos, las complicaciones propias derivadas del avance de la condición son importantes -como ceguera, insuficiencia renal, úlceras en los pies, amputaciones y problemas cardiovasculares relevantes, entre otros-, ya que conllevan a una disminución de la fuerza laboral del país, así como a un aumento del gasto sanitario difícil de cubrir.

Asimismo, los diabéticos con estilos de vida no saludables utilizan con menor frecuencia los recursos de satisfacción consigo mismo, firmeza, perseverancia en la toma de decisiones y autocontrol de las emociones; y predomina en ellos la ansiedad y depresión (Naranjo, López \& Valladares, 2016). Por tanto, la adhesión al tratamiento, en conjunto con otras medidas, podría configurarse en uno de los abordajes menos costosos -desde el punto de vista financiero y sanitario- y más efectivos para el control de la condición, el cual permitiría que la persona también se sienta involucrada y responsable de su tratamiento y, por tanto, obtenga así un mayor bienestar físico, psicológico y social.

Finalmente, es importante plantear algunas implicaciones conceptuales, metodológicas y tecnológicas relacionadas con este campo de investigación. Con respecto a las implicaciones conceptuales, destaca el desarrollo de un posible modelo de abordaje referencial unificador, ya que el modelo biopsicosocial, en el que se enmarca este trabajo, es muy amplio y constituye una perspectiva, mas no un modelo teórico a contrastar con los hallazgos. Esto, por supuesto, conlleva a consideraciones sobre el marco teórico y la definición de las variables dentro de ese enfoque. Posiblemente, esto conduzca a la propuesta de explicaciones más cercanas al fenómeno de las variables relacionadas con la diabetes desde el punto de vista físico, psicológico y social.

En las implicaciones metodológicas se plantea la necesidad de realizar estudios más de índole longitudinal que transversal, con la finalidad de observar cómo se comportan en el tiempo las variables consideradas en este estudio. Por otra parte, una de las limitaciones de esta investigación fue que el tipo de muestreo empleado fue "no probabilístico de tipo intencional", lo que dificulta la posibilidad de generalización de los hallazgos a la población. Es por esto que se recomienda emplear un muestreo de tipo probabilístico en próximas investigaciones. Asimismo, sería importante realizar investigaciones de tipo experimental para corroborar las relaciones entre las variables.

Finalmente, como implicaciones tecnológicas, una de las variables que resalta forzosamente al abordar la condición de la diabetes es la calidad de vida en salud, ya que, según la Federación Internacional de la Diabetes (2011), la diabetes es una enfermedad crónica que afecta la calidad de vida de quienes la padecen, al generar grandes gastos económicos en los países, así como un gran índice de mortalidad. Por tanto, es indispensable llevar a cabo investigaciones que pongan de relieve las variables psicológicas relacionadas con esta condición -estilos de comunicación, adhesión al tratamiento, sexo, edad y apoyo social, entre otras-, y desarrollar programas dirigidos al mejoramiento de la calidad de vida de estas personas.

\section{REFERENCIAS}

Agudelo, D., Buela, G., \& Spolberger, C. (2007). Ansiedad y depresión: el problema de la diferenciación a través de los síntomas. Salud mental, 30(2), 33-41. Recuperado de http:// www.redalyc.org/articulo.oa?id=58230205

Agudelo, D., Casadiegos, C., \& Sánchez, D. (2009). Relación entre esquemas maladaptativos tempranos y características de ansiedad y depresión en estudiantes universitarios. Universitas psychologica, 8(1), 87-104. Recuperado de http:// www.scielo.org.co/pdf/rups/v8n1/v8n1a7.pdf

Aguilar-Salinas, C., Gómez, R., \& Gómez, F. (2011). La diabetes tipo 2 en México: Principales retos y posibles soluciones. Revista de la asociación latinoamericana de diabetes, 19(4), 148-161.

Angelucci, L. (2007). Análisis de rutas: conceptos básicos. Analogías del comportamiento, 10(1), 31-59.

Arancibia, M., Behar, R., Marín, S., Inzunza, N., \& Madrid, E. (2016). Funcionamiento psicosocial en pacientes agudos $\mathrm{y}$ crónicos no psiquiátricos en régimen hospitalario: depresión, alexitimia y falta de asertividad. Revista médica de Chile, 144, 1424-1431. Recuperado de http://www.scielo. $\mathrm{cl} / \mathrm{pdf} / \mathrm{rmc} / \mathrm{v} 144 \mathrm{n} 11 / \mathrm{art08} . \mathrm{pdf}$ 
Asociación Latinoamericana de Diabetes (2013). Guías ALAD sobre el diagnóstico, control y tratamiento de la diabetes mellitus tipo 2 con medicina basada en evidencia. Revista de la ALAD1, 24-28. Recuperado de https://issuu.com/aladdiabetes/docs/guias_alad_2013

Azzollini, S., Bail, V., \& Vidal, V. (2012). El apoyo social y el autocuidado en diabetes tipo 2. Anuario de investigaciones, 19(1), 109-113. Recuperado de http://www.scielo.org. ar/scielo.php?pid $=\mathrm{S} 185116862012000100010 \&$ script $=\mathrm{s}$ ci_arttext.

Benítez, A., Gonzáles, L., Bueno, E., Agüero, F., Alsina, S., Melgarejo, M., Ramírez, A., \& Vera-Ruffinelli, J. (2011). Depresión y Diabetes. Revista ALAD, 19(4), 162-169.

Braz, J., Silva, M., Gois, C., Braz, T., Santos V., \& Silva, L. (2012). Sintomas depressivos e adesâo ao tratamento entre pessoas com diabetes mellitus tipo 2. Revista da rede de emfermagem do nordeste, 13(5), 1092-1099. Recuperado de http://webcache.googleusercontent. com/search?q=cache:PBAJk0EMi8J:www.revistarene. ufc.br/revista/index.php/revista/article/download/65/ $\mathrm{pdf}+\& \mathrm{~cd}=2 \& \mathrm{hl}=\mathrm{es}-419 \& \mathrm{ct}=\mathrm{clnk} \& \mathrm{gl}=\mathrm{ve}$

Calderón, G., Vargas, R., \& Lozano, K. (2015). Tiempo de evolución de diabetes mellitus tipo 2 como factor predisponente de amputación en pacientes con pie diabético. Acta Médica Orreguiana Hampi Runa, 14(1), 25-36. Recuperado de http://www.upao.edu.pe/upload/publicaciones/ hampi_runa/HAMPI_14_1_2014.pdf

Camacho, L., Lucero, L., Agazzi, B., Fernández, A., \& Ferreira, A. (2013) Adherencia al tratamiento en adolescentes con diabetes tipo 1: un enfoque desde la perspectiva de los actores. Enfermería, 1(3), 169-178.

Camejo, M., García, A., Rodríguez, E., Carrizales, M., \& Chique, J. (2012). Visión epidemiológica de la Diabetes Mellitus. Situación en Venezuela. Registro Epidemiológico y propuesta de registro. Programas de detección precoz. Revista venezolana de endocrinología y metabolismo 10(1), 1-6. Recuperado de: http://www.scielo.org.ve/scielo. php?script=sci_arttext\&pid=S1690-3110201200 0400002

Campayo, A., De Jonge, P., Roy, J., Saz, P., De la Cámara, C., ... Quintanilla, M. (2010). Depressive disorder and incident diabetes mellitus: The effect of characteristics of depression. The american journal of psychiatry, 167(5), 580-588.

Cardozo, I., Guarino, L., \& Rondón, J. (2016). Validación del Cuestionario Tridimensional para la Depresión (CTD) en población venezolana con enfermedades crónicas [versión electrónica], Revista de psicopatología y psicología clínica, 21(3), 191-200.

Castaños, C., Reyes, L., Rivera, A., \& Díaz, L. (2011). Estandarización del inventario de asertividad de Gambrill y RicheyII. Revista iberoamericana de diagnóstico y evaluación psicológica, 29(1), 27-50. Recuperado de http://www.aidep. org/03_ridep/R29/r29art2.pdf
Colunga-Rodríguez, C., García, J., Salazar-Estrada, J., \& Angel-González, M. (2008). Diabetes tipo 2 y depresión en Guadalajara, México. 2005. Revista salud pública, 10(1), 137-149. Recuperado de http://www.scielo.org.co/scielo. php?script=sci_arttext\&pid=S0124-00642008000100013

Constantino-Cerna, A., Bocanegra-Malca, M., León-Jiménez, F., \& Díaz-Velez, C. (2014). Frecuencia de depresión y ansiedad en pacientes con diabetes tipo 2 atendidos en un hospital general de Chiclayo. Revista médica herediana, 25(4), 196-210. Recuperado de http://www.scielo.org.pe/scielo. php?script=sci_arttext\&pid=S1018-130X2014000400003

Dalzochio, T., Bonho, L., Feksa, L., \& Berlese, D. (2014) A link between depression and diabetes mellitus. Revista de ciências médicas, 23, 91-99.

De Jonge, P., Alonso, J., Stein, D., Kiejna, A., Aguilar-Gaxiola, S., ... Viana, M. (2014). Associations between DSM-IV mental disorders and diabetes mellitus: a role for impulse control disorders and depression. Diabetologia, 57(6), 699709. Recuperado de https://www.ncbi.nlm.nih.gov/pmc/articles/PMC41249 05/pdf/nihms595266.pdf

De la Cruz-Sánchez, E., Feu, S., \& Vizuete-Carrizona, M. (2012). El nivel educativo como factor asociado al bienestar subjetivo y la salud mental en la población española. Universitas psychologica, 12(1), 31-39. Recuperado de http://revistas.javeriana.edu.co/index.php/revPsycho/article/viewFile/1735/3960

Doyle, T., Halaris, A., \& Rao, M. (2014). Shared neurobiological pathways between type 2 diabetes and depressive symptoms: A review of morphological and neurocognitive findings. Current diabetes reports, 14(560), 2-12. doi:10.1007/ s11892-014-0560-7

Escandón, N., Azócar, B., Pérez, C., \& Matus, V. (2015). Adherencia al tratamiento en diabetes tipo 2: su relación con calidad de vida y depresión. Revista de psicoterapia, 26(101), 125-138. Recuperado de http://www.revistadepsicoterapia. com/ojs/index.php/rdp/article/view/31

Fabián, M., García, M., \& Cobo, C. (2010). Prevalencia de síntomas de ansiedad y depresión en pacientes con diabetes mellitus tipo 2 y su asociación con el tipo de tratamiento, complicaciones de la diabetes y comorbilidades. Interna de Mexico, 26(2), 100-108. Recuperado de http://cmim.org/ boletin/pdf2010/M edIntContenido02_05.pdf

Federación Internacional de la Diabetes (2011). Plan Mundial contra la Diabetes 2011-2021. Bruselas: Autor.

Garay, M., Santiago, J., \& Malaraca, J. (2011). Estrategias de afrontamiento y adherencia al tratamiento en pacientes con diabetes mellitus tipo 2. Revista de investigación clínica, 63(2), 151-161.

García, E., \& Magaz, Á. (2000). ADCAs autoinformes de actitudes y valores en las interacciones sociales: manual de referencia. Barakaldo: ALBOR-COHS.

Gomes-Villas, L., Foss, M., Foss de Freitas, M., \& Pace, A. (2012). Relación entre apoyo social, adhesión al tratamiento y control metabólico en personas con diabetes mellitus. 
Revista latinoamericana de emfermagem, 20(1), 52-58. Recuperado de http://www.scielo.br/pdf/rlae/v20n1/es_08. pdf.

González, A., Gilbaja, L., Villa, E., Acevedo, O., Ramírez, M., Ponce, E. (2015). Niveles de adherencia al tratamiento y el control glucémico en pacientes con diabetes mellitus tipo 2 descontrolados. Revista mexicana de endocrinología, metabolismo y nutrición, 2, 11-17.

Hermanns, N., Ehrmann, D., \& Kulzer, B. (2015). How should we treat people with diabetes and comorbid depression? Journal of diabetes and its complications, 30, 187-188.

Hernández, R., Fernández, C., \& Baptista, P. (2006). Metodología de la investigación. México: McGraw-Hill.

Centros para el Control y la Prevención de Enfermedades (2017). Informe Nacional de Estadísticas de la Diabetes (2017). Recuperado de https://www.cdc.gov/diabetes/pdfs/ data/statistics/national-diabetes-statistics-report-spanish. pdf.

Instituto Nacional de Estadísticas. (2014). Boletín indicadores educativos derivados de la encuesta de hogares por muestreo. Caracas: Instituto Nacional de Estadísticas.

Jiménez, G., \& Miguel-Tobal, J. (2003). El cuestionario tridimensional para la depresión (ctd): Un nuevo instrumento para medir la tristeza/depresión. Ansiedad y estrés, 9(1), 17-34.

Kaltman, S., Serrano, A., Talisman, N., Magee, M., Cabassa, L, Pulgar-Vidal, O. (2016). Type 2 diabetes and depression. A pilot trial of an integrated self-management intervention for latino immigrants. The diabetes educator, 42(1), 87-95.

Kerlinger, F., \& Lee, H. (2002). Investigación del comportamiento. Métodos de investigación en ciencias sociales. México: McGraw Hill.

Ledón, L. (2012). Impacto psicosocial de la diabetes mellitus, experiencias, significados y respuestas a la enfermedad. Revista cubana endocrinológica, 23(1), 76-97.

Ledón, L. (2014). La comunicación en el campo de la diabetes mellitus, algunas pautas para su acción efectiva. Revista cubana endocrinológica, 25(2), 124-138.

Lyketsos, C. (2010). Depression and Diabetes: More on what the relationship might be. The american journal of psychiatry, 167(5), 498-500. Recuperado de http://ajp.psychiatryonline.org/doi/full/10.1 176/appi.ajp.2010.10020243

López-Carmona, J., Arisa-Andraca, C., Rodríguez-Moctezuma, J., \& Munguia-Miranda, C. (2003). Construcción y validación inicial de un instrumento para medir el estilo de vida en pacientes con diabetes mellitus tipo 2. Salud pública mexicana, 45(4), 259-268. Recuperado de http://www.scielo. org.mx/pd f/spm/v45n4/a04v45n4.pdf

Maidana, G., Lugo, G., Vera, Z., Acosta, P., Morinigo, M., ... Isasi, D. (2016). Factores que determinan la falta de adherencia de pacientes diabéticos a la terapia medicamentosa. Memorias del instituto de investigaciones en ciencias de la salud, 14(1), 70-77.
Marco, A. (2014). Propuesta de mejora educativa para pacientes diabéticos tipo 2 (trabajo de grado en enfermería no publicado). Universidad Pública de Navarra, España.

Marín, M., \& Mubayed, J. (2012). Factores psicosociales asociados a la adherencia al tratamiento en pacientes con diabetes mellitus. Un modelo de ruta. (trabajo especial de grado no publicado). Universidad Centro Occidental "Lisandro Alvarado", Barquisimento, Venezuela.

Martínez, M., Justicia, F., \& Fernández, E. (2016) El papel de la asertividad docente en el desarrollo de la competencia social de su alumnado. Electronic journal of research in educational psychology, 14(39), 310-332.

Méndez, F., \& Beléndez, M. (1994). Variables emocionales implicadas en el control de la diabetes: estrategias de intervención. Anales de psicología, 10(2), 189-198. Recuperado de https://rua.ua.es/dspace/bitstream/10045/15379/1/Mendez_Belendez_Variables_emocionales.pdf

Ministerio del Poder Popular Para la Salud. (2014). Anuario de mortalidad 2014. Caracas: Autor.

Molina, A., Acevedo, O., Yáñez, M., Dávila, R., \& González, A. (2013). Comparación de las prevalencias de duelo, depresión y calidad de vida asociados con la enfermedad entre pacientes con diabetes mellitus tipo 2 descontrolados y controlados. Revista de especialidades médico-quirúrgicas, 18(1), 13-18. Recuperado de http://www.medigraphic.com/ pdfs/quirurgicas/rmq-2013/rmq131c.pdf

Moral, J., \& Cerda, M. (2015). Predictores psicosociales de la adherencia a la medicación en pacientes con diabetes tipo 2. Revista iberoamericana de psicología y salud, 6(1), 1927. Recuperado de https://www.sciencedirect.com/science/ article/pii/S2171206915700037.

Moscoso, M. (2014). Naturaleza de las emociones positivas en la evaluación de la depresión: una nueva visión en psicometría. Revista de psicología, 32(2), 304-327. Recuperado de http://revistas.pucp.edu.pe/index.php/psicologia/article/ view/10951/11461

Nagel, N., Azócar, B., Pérez, C., \& Matus, V. (2015). Adherencia al tratamiento en diabetes tipo 2: Su relación con Calidad de Vida y Depresión. Revista de psicoterapia, 26(101), 125-138.

Naranjo, Y., López, L., \& Valladares, A. (2016). Enfoque psicológico del estilo de vida de los adultos medios diabéticos tipo II. Revista cubana de medicina integral general, 35(4). Recuperado de http://www.revmgi.sld.cu/index.php/mgi/ article/view/210/102

Oblitas, L. (2004). Psicología de la salud y calidad de vida. México: Thomson.

Oliveira, V., \& Trujillo, S. (2017). Autocuidado y adherencia al tratamiento en pacientes con diabetes mellitus del servicio de medicina interna del hospital universitario Dr. Ángel Larralde. Medicina interna, 33(1), 24-34.

Organización Mundial de la Salud (2014) Estadísticas sanitarias mundiales 2014. Ginebra: OMS. 
Organización Panamericana de la Salud. (2016). Día Mundial de la Salud 2016: diabetes. Recuperado de http://www. who.int/campaigns/world-health-day/2016/event/es/

Ortega, M., \& Calero, A. (2015). Un caso de interacción no asertiva. Clínica contemporánea, 6(1), 21-32. Recuperado de http://www.copmadrid.org/webcopm/publicaciones/clinicacontemporanea/cc2015v6n1a3.pdf

Ortiz, M., \& Myers, H. (2014). Control metabólico en pacientes diabéticos tipo 1 chilenos: rol del estrés psicológico. Revista médica de Chile, 142(4), 451-457. Recuperado de http:// www.scielo.cl/pdf/rmc/v142n4/art06.pdf

Ortiz, M., Baeza-Rivera, M., \& Myers, H. (2013). Propiedades psicométricas de la escala de estrés para diabéticos en una muestra de pacientes diabéticos tipo II chilenos. Terapia psicológica, 31(3), 281-286. Recuperado de http://www. scielo.cl/pdf/terpsicol/v31n3/art02.pdf

Páez, A., Jofré, M., Azpiroz, C., \& De Bortoli, M. (2009). Ansiedad y depresión en pacientes con insuficiencia renal crónica en tratamiento de diálisis [versión electrónica], Universitas psychologica, 8(1), 117-124. Recuperado de http://www.scielo.org.co/scielo.php?script=sci_arttext\&pid $=$ S1657-92672009000100009

Patilla, C. (2011). Ansiedad, depresión y situaciones generadoras de estrés en pacientes diabéticos tipo 2 con adherencia $y$ sin adherencia al tratamiento (trabajo especial de grado no publicado). Universidad Nacional Mayor de San Marcos, Lima, Perú.

Pérez, E., Bautista, S., \& Bazaldúa, A. (2008). Susceptibilidad percibida de diabetes mellitus en adultos no diagnosticados. Recuperado de http://www.psicologiacientifica.com/ bv/psicologia-317-1-susceptibilidad-percibida-de-diabetesmellitus-en-adultos-no.html.

Pineda, N., Bermúdez, V., Cano, C., Ambard, M., Mengual, E., Medina, M., ... Leal, E. (2004) Aspectos Psicológicos y Personales en el manejo de la Diabetes Mellitus. Archivos venezolanos de farmacología y terapéutica, 23(1), 13-17. Recuperado de http://www.scielo.org.ve/scielo.php?script=sci_art text\&pid=S079802642004000100003\&lng=es\&tlng=es.

Ponce, J., Velázquez, A., Márquez, E., López, L., \& Bellido, M. (2009). Influencia del apoyo social en el control de las personas con diabetes. Index de Enfermería, 18(4), 224-228. Recuperado de http://scielo.isciii.es/scielo.php?script=sci arttext\&pid=S1132-12962009000400002

Ramos, L., \& Arnaud, E. (2011). Emotional factors, life quality and adhesion of treatment in adult with type 2 diabetes. Journal of human growth and development, 21(3), 867-877. Recuperado de http://www.revistas.usp.br/jhgd/article/ viewFile/20039/22138

Ricard, F. (2008) Tratado de osteopatía visceral y medicina interna. Sistema digestivo (Tomo 2). Madrid: Editorial Médica Panamericana.

Rodrígues, E., Egidio, A., \& Cardoso, A. (2014). The Role of Depression on Glycemic Control. Journal of endocrinology metabolism, 4, 119-120.
Rodríguez, M., \& García, J. (2013). Efectos de una intervención psicológica en los niveles de glucosa en pacientes con diabetes tipo 2. Revista electrónica de psicología - Iztacala, 16(4), 1262-12. Recuperado de http://www.iztacala. unam.mx/carreras/psicologia/psiclin/vol16num4/Vol16No4Art10.pdf

Rondón, J. (2011). Variables psicosociales implicadas en el mantenimiento y control de la diabetes mellitus: investigaciones y hallazgos. Revista electrónica de psicología - Iztacala, 14(2), 126-161. Recuperado de http://www.revistas. unam.mx/index.php/repi/article/viewFile/26030/24506

Rondón, J. (2015). Variables biopsicosociales que discriminan el tipo de tratamiento farmacológico en la diabetes mellitus tipo 2 (trabajo de ascenso no publicado). Universidad Central de Venezuela, Caracas.

Rondón, J., Cardozo, I., \& Lacasella, R. (2014). Depresión, estilo de comunicación pasivo-asertivo-agresivo y adhesión al tratamiento en paciente con diabetes mellitus tipo 2. (manuscrito no publicado). Instituto de Psicología, Universidad Central de Venezuela, Caracas.

Rondón, J., \& Lugli, Z. (2013). Efectos del tipo de diabetes, estrategias de afrontamiento, sexo y optimismo en el apoyo social. Revista de Psicopatología y Psicología Clínica, 18(3), 193-207.

Sánchez-Cruz, J., Hipólito-Lóenzo, A., Mugártegui-Sánchez, S., \& Yáñez-González, R. (2016). Estrés y Depresión asociados a la no adherencia al tratamiento en pacientes con Diabetes Mellitus tipo 2. Atención familiar, 23(2). Recuperado de http://dx.doi.org/10.1016/j.af.2016.03.003

Santos, C., De Oliveira, E., \& Sardinha, A. (2012). Deficiências em habilidades sociais na depressao: estudo comparativo. Psicologia: Teoria e Prática, 14(1), 183-196.

Saur, C., \& Steffens, D. (2010). Adherence to treatment for depression. En H. Bosworth (ed), Improving patient treatment adherence: A clinician's guide (pp 241-257). USA: Springer.

Schmitt, A., Reimer, A., Kulzer, B., Haak, T., Gahr, A., \& Hermanns, N. (2015) Negative association between depression and diabetes control only when accompanied by diabetesspecific distress. Journal of behavioral medicine, 38, 556564.

Seligman, M. (1975). Indefensión. Madrid: Titivillos.

Stuart, G., \& Laraia, M. (2006). Enfermería psiquiátrica: principios y práctica (8 ed). Barcelona, España: Elsevier.

Valls, A. (2009). Cómo disponer de más tiempo. Guía práctica para superar los mayores peligros en la gestión de su tiempo. Barcelona, España: Profit editorial.

Young-Hyman, D., De Groot, M., Hill-Briggs, F., González, J., Hood, K., \& Peyrot, M. (2016). Psychosocial care for people with diabetes: A position statement of the American Diabetes Association. Diabetes care, 39, 2126-2140. 